\title{
AN ANALYTICAL MODEL FOR A SLOTTED WDM METRO RING WITH A-POSTERIORI ACCESS
}

\author{
Kemal Bengi \\ Institute of Communication Networks, Vienna University of Technology, Favoritenstrasse 9/388, \\ A-1040 Vienna, Austria \\ kemal.bengi@tuwien.ac.at
}

\begin{abstract}
In this paper, an analytical model based on the semi-Markov theory is developed to quantify the performance of a distributed access protocol for a slotted WDM metro ring employing all-optical packet switching and based on an a-posteriori packet selection strategy. In our architecture, each node is equipped with one tunable transmitter and one fixed-tuned receiver. Furthermore, as we generally consider a-posteriori access strategies, different packet selection schemes are compared by simulations.
\end{abstract}

Keywords: All-optical ring networks, wavelength division multiplexing (WDM), medium access control (MAC) protocols, semi-Markov analysis

\section{Introduction}

In order to eliminate or, at least, alleviate the electronic bottleneck encountered in current optical networks, all-optical packet switching solutions have to be deployed in future photonic networks. In the local and metropolitan area (LAN/MAN) environment, all-optical packet switching can be already achieved with current optical technology. Particularly, metropolitan optical WDM networks are rapidly emerging as viable and cost-effective solutions for many telecommunication carriers and service providers who have to cope with an extremely growing bandwidth demand and expensive additional fiber installation costs. In fact, optical packet-switched WDM ring network prototypes are being built and experimentally studied in several research laboratories, e.g. [12, 5]. In order to reduce the hardware costs and improve the node scalability associated with employing transceiver arrays (with as many transmitters and receivers as there are accommodated nodes and/or channels) at each network node, different node structures have to be envisaged also in the WDM ring case. One applicable approach is to deploy one fixed-tuned optical transmitter and one fixed-tuned optical receiver (TT-FR system). This architecture may be preferred over its 
FT-TR counterpart, due the recent advances in the technology of fast-tunable optical transmitters which typically are by far easier to implement than rapidly tunable receivers.

Generally, in order to avoid collisions on the individual WDM channels of such networks, MAC (Medium Access Control) protocols are necessary to arbitrate the channel access. Several access protocols for all-optical slotted WDM rings have been proposed in the literature $[8,5,6,7]$. Most of them are based on the case, where there are as many wavelength channels as nodes in the network, which results in serious scalability problems, especially in metro networks. Moreover, some proposals require transmitter and/or receiver arrays at each node associated with high equipment costs.

In this paper, an analytical model based on the semi-Markov process methodology is developed to quantify the performance of an access protocol for a slotted WDM metro ring. The considered system employs all-optical packet switching and supports much more nodes than there are available wavelengths in the network. Furthermore, the network in question is based on time-slotted WDM channels and relies on optical add/drop multiplexers at each node. Since one channel is shared by several destination nodes, we obtain a multi-hop WDM ring as underlying network architecture. The channels are divided into several slots allowing data packets to be transmitted and received in a highly bandwidthefficient way. The required signaling information, i.e., the destination address and the channel/slot availability status, may be provided via the already mature subcarrier multiplexing (SCM) technique [4]. Furthermore, as we generally consider the so-called a-posteriori access strategy [3], where the corresponding data packet is selected from the appropriate transmission buffer after having detected the signaling information, different packet/buffer selection schemes may be proposed and compared [2].

The remaining part of this paper is organized as follows. In Section 2, the network architecture along with the node structure and the considered signaling technique is presented. Section 3 describes the general considered medium access strategy based on the random packet selection scheme for the WDM slotted ring. In Section 4, a performance study is carried out by means of a semi-Markov model and computer simulations. Finally, Section 5 provides some conclusions.

\section{Network Architecture}

The considered system is based on a ring topology interconnecting $M$ nodes via a single unidirectional optical fiber, as shown in Fig. 1. The bandwidth of the fiber is divided into multiple wavelength channels $\left\{\lambda_{1}, \lambda_{2}, \ldots, \lambda_{C}\right\}$, which in turn are divided into fixed-length time slots, constituting a slotted WDM ring with $C$ data channels. Each slot consists of a header and a payload field. 
One data packet is considered to fit exactly into the slot's payload section. The slots are continuously circulating on the ring and can be empty or full. It is assumed that the slots are perfectly synchronized between the channels, though a slight time skewing (e.g., due to dispersion effects) will not negatively affect the proper operation of the access protocol presented below. Due to the support of multiple channels on the ring, one single channel may operate at a typical electronic transmission speed, e.g. $2.5 \mathrm{Gbit} / \mathrm{s}$, while the aggregate bandwidth is equal to a multiple of the channel bit-rate, potentially enabling Tbit/s throughputs.

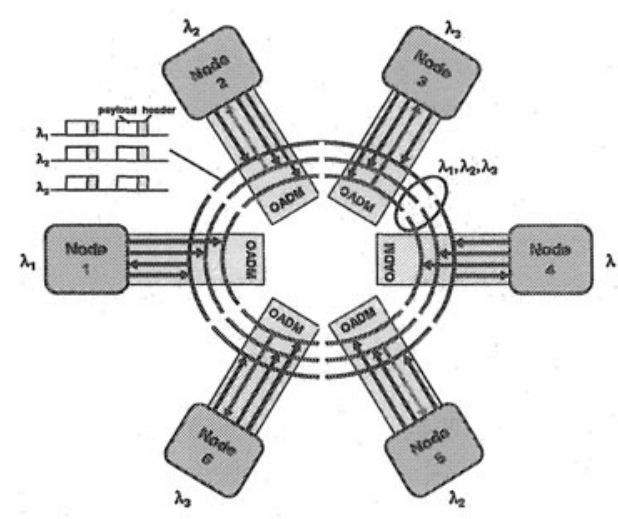

Figure 1. Time-slotted WDM metro ring $(M=6, C=3)$

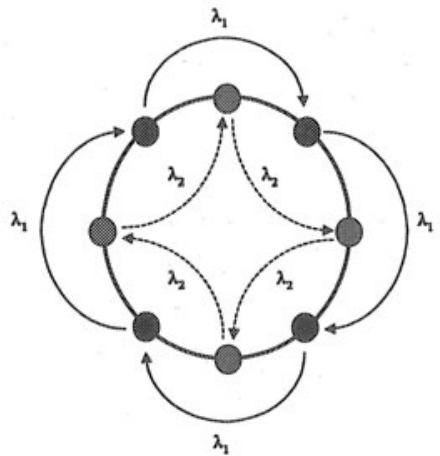

Figure 2. Conceptual multi-hop WDM ring $(M=8, C=2)$

In our architecture (Fig. 1), each network node is equipped with only one tunable transmitter and one fixed-tuned receiver, referred to as TT-FR system. Since the transmission and reception of data packets is performed all-optically, the nodes may be viewed as optical add/drop multiplexers (OADM). Consequently, a node may send packets on any wavelength available in the network, while it is allowed to receive packets only on a preassigned wavelength (drop channel). As a result, no inherent scalability problems arise with such an architecture as opposed to systems deploying transceiver arrays, which steadily have to be adapted to the number of supported WDM channels.

It is further assumed that there are more network nodes than wavelengths in the system, i.e. $M>C$, which significantly relaxes the scalability constraint encountered in systems where $M=C$, since the channel number is decoupled from the number of nodes. For symmetry considerations, $M$ is assumed to be an integer multiple of the number of channels $C$. We further suppose that the $C$ wavelengths are assigned in a cyclic (interleaved) fashion to the $M$ destination nodes, as demonstrated in Fig. 1 and Fig. 2. Thus, several nodes have to use 
the same channel for the reception of packets. When $D$ denotes the number of nodes and channels ratio, i.e. $D=M / C$ being an integer, exactly $D$ nodes have to share one drop channel. Formally, the destination nodes $d=c+n \cdot C$ with $c \in\{1,2, \ldots, C\}, n \in\{0,1, \ldots, D-1\}$ and $d \in\{1,2, \ldots, M\}$ share the same channel $i$ for packet reception. Since we additionally assume destination release and slot reuse of the transmitted packets, one slot of a channel may be filled and released several (in maximum $D$ ) times within one ring latency, yielding an improved degree of statistical multiplexing and bandwidth efficiency within the ring. As a result, multihopping is introduced in the considered architecture, illustrated in Fig. 2 for 8 nodes and 2 channels.

However, compared to the case where each destination node is preallocated a unique wavelength $(M=C)$, the contention in accessing the slots of one channel is significantly increased leading to more congestion on the ring. Under these circumstances, the alleviation of the head-of-line (HOL) blocking problem observed in the source node's transmission queue becomes a very crucial issue. Therefore, in order to alleviate the HOL blocking, one separate queue is assigned to each channel available in the network, giving totally $C$ buffers at every source node which are represented by $b=\left\{b_{1}, b_{2}, \ldots, b_{C}\right\}$. The allocation of a data packet to be transmitted to one of the $C$ buffers $b_{c}, c \in\{1,2, \ldots, C\}$ is determined according to $c=d \bmod C$, if $d / C$ is not an integer, and $c=C$ otherwise (i.e., if $d \bmod C=0$ ), where $d \in\{1,2, \ldots, M\}$ represents the destination node index associated with the current packet to be stored.

\section{Access Control Strategy}

The considered access protocol for the slotted WDM metro ring is mainly related to the buffer selection scheme for packet transmission at a slot time (since we consider only one transmitter at each node). According to the generic slotted ring access mechanism, slots are continuously circulating around the ring on each channel with the slot availability being indicated by the SCM header. When an arriving slot is empty, the node can use this slot to transmit its packet, otherwise it has to wait until an empty slot arrives. This way we have no channel and receiver collisions, as well as no packet losses by using this basic access strategy. As mentioned above, destination release and slot reuse is employed in our WDM metro ring. Thus, slots can be reused a number of times during the ring latency.

In principle, the so-called a-posteriori packet selection strategy is considered throughout this paper. This means, that first the availability status of the wavelength channels at a slot time is detected by means of the SCM signaling technique (building a channel availability vector), and then the appropriate packet is selected from the corresponding transmission queue at the source node for transmission in the current slot. The packet selection is necessary since only 
one packet may be transmitted per slot time due to the underlying node structure (TT-FR).

Generally, each empty slot on any channel can be used for packet transmission by a source node. However, when more than one channel carries an empty slot in the current slot period, one packet (or equivalently, one buffer) corresponding to one of the empty channels has to be chosen according to a certain selection rule. As a consequence, the a-posteriori packet selection process has to be performed at a higher speed in the electronic domain, which slightly increases the processing complexity compared to an a-priori packet selection scheme. Upon arrival of a new slot, each source node detects the slot availability on each channel indicated by the SCM header and establishes the channel availability vector $c_{a}$ made up of maximally $C$ elements (when all channels are empty in the current slot). For instance, when channels $\lambda_{i}, \lambda_{j}$, and $\lambda_{k}$ are currently free, then $c_{a}=\{i, j, k\}$ with $\left|\mathfrak{c}_{a}\right|=3$. If no empty slot is available, the node has to wait for the next slot, otherwise it checks whether there are more than one free slot available. When just one slot is free, the node simply transmits the packet from the transmission queue corresponding to the empty channel. In the case of several available channels, i.e. $\left|c_{a}\right|>1$, a specific packet or buffer selection method has to be applied. Different packet and buffer selection strategies may be envisaged at each sending node for the case that more than one slot is free at a slot instant (referred to as buffer contention), namely the random (RND), longest queue (LQ), round robin (RR), maximum hop (MH), and C-TDMA selection schemes. These are discussed and compared in [1]. For the following, let $\boldsymbol{b}_{s}=\left\{\boldsymbol{b} \mid b_{c}\right.$ is active and channel $c$ is empty $\}$, hereafter called as buffer preselection set.

\section{Performance Analysis}

In the following subsection, we provide an analytical model for analyzing the RND access strategy for uniform Poisson traffic with fixed-length data packets, whereas Subsection 4.3 evaluates the performance of all the proposed buffer selection schemes by computer simulations under more realistic traffic conditions.

\subsection{Analytical Model}

In the literature, some analytical approaches for studying a slotted WDM ring have been developed so far, e.g. [9, 10]. However, to our knowledge, a-posteriori access schemes with $M>C$ are analytically evaluated for the first time. The approximate analytical performance evaluation in this paper is based on a semi-Markov model [11]. Semi-markov processes may accommodate arbitrary packet interarrival times as well as arbitrarily distributed state sojoum times. The goal of the developed semi-Markov model is to determine the 
channel throughputs and the mean queueing delays of the considered access protocol.

Model Assumptions. The assumptions for the semi-Markov analysis of the underlying RND access protocol can be summarized as follows.

(1) All network nodes operate equally and independently.

(2) The system time is normalized to slots.

(3) Data packets have fixed lengths equaling one slot $(L=1)$.

(4) Each node generates data packets according to a Poisson process with mean arrival rate $\lambda$.

(5) A node may generate in maximum one data packet per slot.

(6) The destination addresses of the generated data packets are uniformly distributed among the network nodes (assuming that a source node cannot transmit to itself).

(7) Only transmissions on a single channel implying a single transmission buffer at one node is considered here (however, capturing the interdependencies between the transmission dynamics of all channels).

(8) The transmission queue size $B$ (related to one channel) of the nodes is finite, i.e. $B<\infty$.

Herein, a single node may be taken to be typical of the behavior of each node in the system. Consequently, the protocol behavior can be appropriately described by the operation states of a source node, i.e., in this case, the states of the tunable transmitter which is tunable to any of the $C$ channels for packet transmission.

Global States and State Diagram. The possible states in which a source node of the considered slotted WDM ring can reside in terms of a certain channel are listed below.

idle $(0)$ :

- wait for the generation of a new data packet

synchronization (1):- wait for the next slot header when a data packet is ready for transmission

evaluation (2): - detect and evaluate the slot header

delay (3): - wait for the next slot due to blocking 
transmission (4): - transmit the corresponding data packet

The related state diagram in Figure 3 reflects the defined source node states differentiated by different columns (global states), and the transmission queue load given as the number of currently buffered packets at the source node (local states) in $B+1$ rows. From Figure 3, it can be seen that the state space cardinality of the developed semi-Markov chain is equal to $4(B+1)+1$.

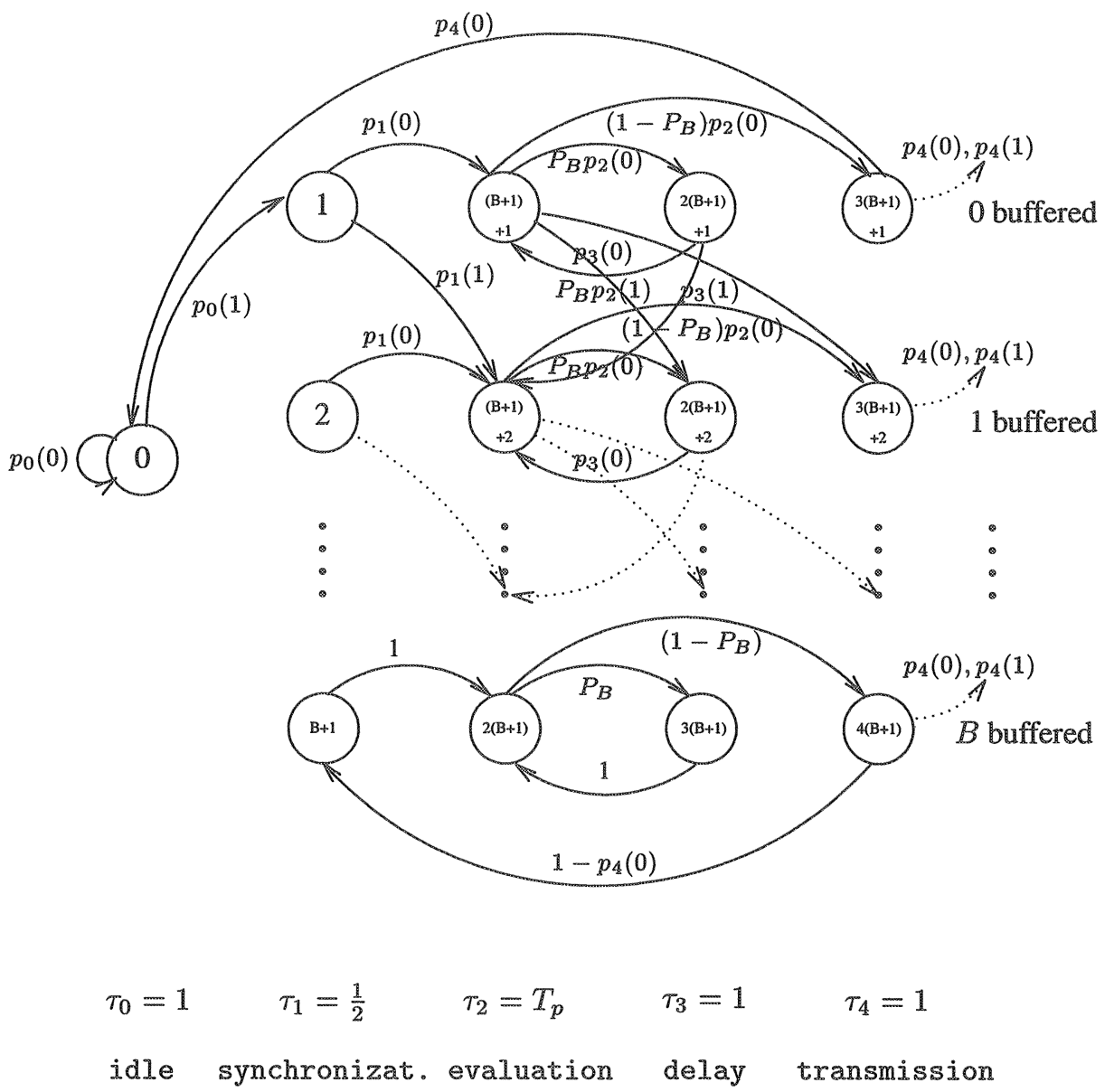

Figure 3. State diagram for the slotted WDM metro ring relying on a RND a-posteriori access strategy with uniform traffic.

Throughput and Delay。 Let $p_{i}, i \in\{0,1, \ldots, 4(B+1)\}$ denote the stationary state probabilities of an embedded Markov chain whose time instants are per 
definition identical to the state transition instants in terms of the semi-Markov chain, and $\tau_{i}, i \in\{0,1, \ldots, 4(B+1)\}$ be the state sojourn times of the semiMarkov process, then the stationary state probabilities (limiting probabilities) of the semi-Markov chain, $P_{i}$, are given by the following fundamental relation [11]:

$$
P_{i}=\frac{p_{i} \tau_{i}}{\sum_{j=0}^{4(B+1)} p_{j} \tau_{j}}, \quad i=0,1, \ldots, 4(B+1) .
$$

Note that the state probabilities of the embedded Markov chain can be obtained from $p_{j}=\sum_{i=0}^{4(B+1)} p_{i} p_{i, j}, j \in\{0,1, \ldots, 4(B+1)\}$, where $p_{i, j}$ represents the corresponding transition probabilities. Furthermore, the probability $l_{i}, i \in$ $\{0,1, \ldots, 4(B+1)\}$ that state $i$ will be left in the current slot is given by $l_{i}=P_{i} / \tau_{i}$.

The individual transition probabilities $p_{i, j}(i, j \in\{0,1, \ldots, 4(B+1)\})$ of the semi-Markov model depend on the respective probabilities that exactly $k$ new data packets are generated during the corresponding sojourn time $\tau_{n}$ $(n=0,1, \ldots 4)$. Considering a Poisson arrival process with mean arrival rate $\lambda$, one directly obtains $p_{n}(k)=\frac{\left(\lambda \tau_{n}\right)^{k}}{k !} e^{-\lambda \tau_{n}}, n \in\{0,1, \ldots 4\}$.

Next, the (source) node throughput $S_{n}$ per channel can be accordingly callculated by $S_{n}=\sum_{i=1}^{B+1} P_{3(B+1)+i}$, which immediately leads to the overall channel throughput $S$ as

$$
S=M \cdot S_{n}=M \cdot \sum_{i=1}^{B+1} P_{3(B+1)+i} .
$$

In order to obtain the mean queueing delays, again, $N$ denotes the total number of data packets waiting for transmission at the source node (considering all the $C$ transmission buffers) in the current slot, thus $E[N]=\sum_{i=0}^{4(B+1)} E\left[N_{i}\right] P_{i}$, from which we obtain the mean packet queueing delay $E\left[d_{q}\right]$ of a source node associated with a single channel, measured from the generation instant of a data packet to the moment of its complete transmission, via Little's Law:

$$
E\left[d_{q}\right]=\frac{1}{C} \cdot \frac{E[N]}{S_{n}} \text {. }
$$

The individual $E\left[N_{i}\right]$ are here given by

$$
E\left[N_{i}\right]=\left\{\begin{array}{cl}
0 & ; \quad i=0 \\
\sum_{j=0}^{B+1-i}\left(i+\frac{1}{2} j\right) \cdot p_{1}(j) ; & 1 \leq i \leq(B+1) \\
i & ; \quad(B+1)+1 \leq i \leq 4(B+1)
\end{array} .\right.
$$


Thus, the mean delay/channel throughput characteristics of the considered protocol can be evaluated by using equations (2) and (3). The crucial measure for the evaluation of the developed semi-Markov model is the blocking probability $P_{B}$. The RND buffer selection strategy and uniform traffic is assumed for the calculation of $P_{B}$ here. In the following modelling approach, we assume that the global and local states of the basic state diagram shown in Figure 3 refer to transmissions on only one channel. That means that the node transmissions on a specific channel implying a single buffer is analyzed and is taken as representative for the other channels' behavior which is justified due to the assumed traffic symmetry.

Blocking Probability. Let $P_{f}$ be the probability that the current slot on a certain channel $c$ is free and $P_{c}$ be the probability that the queue corresponding to this channel, $b_{c}$, is chosen (based on the a-posteriori random selection strategy). Then, the probability that the current slot on a certain channel is accessible by the source node is given by $P_{A}=P_{f} \cdot P_{c}$, and

$$
P_{B}=1-P_{A}=1-P_{f} P_{c}
$$

First, for calculating $P_{f}=P$ \{considered channel is free in the current slot , we divide the ring network into $D$ segments and consider only transmissions on one channel. Different segments depending on the considered channel can be defined (called channel segments), where the first node of the segment may receive on the considered (drop) channel.

Due to the employed destination release in the WDM metro ring, some source nodes possess a higher channel access probability within a segment than others. In this context, different channel access priorities $p$ may be introduced. A source node within a segment, e.g. segment 1 , willing to transmit on a certain channel is blocked when either segment 1 is traversed by traffic from the other $D-1$ segments, which is captured by the probability $P_{\text {inter }}$, or when the node is traversed by traffic from other nodes within the same segment 1 , which is represented by the probability $P_{\text {intra. }}$. With $S_{s, d}=S_{n} /(M-1)$, $s, d \in\{1,2, \ldots, M\}, s \neq d$, denoting the individual source/destination node throughputs and $p$ representing the intrinsic access priorities defined above, based on [9], $P_{\text {inter }}$ is given by

$$
P_{\text {inter }}=S_{s, d}\left[(C-1)(D-1)+\frac{C(D-2)(D-1)}{2}\right],
$$

and $P_{\text {intra }}$ can be obtained from

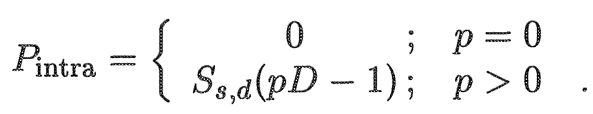


Eventually, we get $P_{f}(p)=1-\left(P_{\text {inter }}+P_{\text {intra }}\right)$ as

$$
P_{f}(p)= \begin{cases}\frac{2-S_{s, d}\left(C D^{2}-D C-2 D+2\right)}{2} ; & p=0 \\ \frac{2-D \cdot S_{s, d}[C(D-1)+2 p-2]}{2} ; & 1 \leq p \leq C-1 .\end{cases}
$$

Since all the access priorities $p$ are equally likely for the source nodes (due to $D$ being an integer) for any segment, $P_{f}$ is obtained from $P_{f}(p)$ by averaging over $C$ and $D$. Eventually, $P_{f}$ is given by

$$
\begin{aligned}
P_{f}= & \frac{1}{D C} \cdot \frac{1}{2}\left\{2-S_{s, d}\left(D^{2} C-D C-2 D+2\right)+\right. \\
& \left.+\sum_{p=1}^{C-1}\left(2-D \cdot S_{s, d}[C(D-1)+2 p-2]\right)\right\} .
\end{aligned}
$$

Concerning the calculation of $P_{c}$, it may be differentiated between two possible RND buffer selection schemes. Firstly, the variant where the random choice of one queue (out of the contending queues) is performed without taking the full/empty status of the contending buffers into account, identified by strategy 1 , and secondly, the variant where the activity of the transmission queues is considered in the selection process (i.e., $b_{s}$ is taken as subset for the selection), indicated by strategy 2 . Note that in both cases the subset of queues associated with free channels is used as basis for the selection.

For calculating $P_{c_{1}}=P$ \{queue $b_{c}$ is selected $\left.\mid b_{c} \in b_{s_{0}}\right\}$, i.e., the probability that a queue is randomly selected out of the set of queues, whose corresponding channels are free at the current slot, and $P_{c_{2}}=P$ \{queue $b_{c}$ is selected $\mid b_{c} \in b_{s}$, i.e., the probability that a queue is randomly selected out of the set of queues, which additionally are active (non-empty) in the current slot, the theorem of total probabilities is to be applied. In terms of determining $P_{c_{1}}$, note that a queue is only preselected (being an element of $b_{s_{0}}$ ) when its allocated channel is free in the current slot time here. Accordingly, the probability that a queue is preselected equals $P_{f}$. As a result, the probability that a specific queue is randomly selected when all the remaining $C-1$ channels are also free is provided by $(1 / C) \cdot P_{f}^{C-1}$, the probability that a queue is selected given that all the other channels except one, i.e. $C-2$ channels, are free is $(1 /(C-1))$. $(C-1) P_{f}^{C-2}\left(1-P_{f}\right)$, and so forth. Generally, we obtain

$$
P_{c_{1}}=\sum_{i=1}^{C} \frac{1}{c}\left(\begin{array}{c}
C-1 \\
c-1
\end{array}\right) P_{f}^{c-1}\left(1-P_{f}\right)^{C-c}
$$

Concerning the calculation of $P_{c_{2}}$, recall that a queue is only preselected (being an element of $\boldsymbol{b}_{s}$ ), if it is non-empty and the corresponding channel is free. 
Consequently, the probability that a queue is preselected is given by $P_{f}^{*}=$ $P_{f} P_{a}$, while the probability that a queue is blocked equals $P_{b}=\left(1-P_{f}\right)(1-$ $\left.P_{a}\right)+P_{f}\left(1-P_{a}\right)$. Further, $P_{a}$, the probability that a queue (excluding the considered one) is active, is readily obtained by including the semi-Markov state probability of being in state idle, i.e. $P_{a}=\left(1-P_{0}\right) /(C-1)$. Finally, $P_{c_{2}}$ is obtained from (10) by $P_{f}$ replacing with $P_{f}^{*}$ and $\left(1-P_{f}\right)$ with $P_{b}$. Thus, the first case, i.e. $P_{c}=P_{c_{1}}$ is determined as

$$
P_{c_{1}}=\sum_{i=1}^{C} \frac{1}{c}\left(\begin{array}{c}
C-1 \\
c-1
\end{array}\right) P_{f}^{c-1}\left(1-P_{f}\right)^{C-c}
$$

and the second case, i.e. $P_{c}=P_{c_{2}}$ is given by

$$
P_{c_{2}}=\sum_{i=1}^{C} \frac{1}{c}\left(\begin{array}{c}
C-1 \\
c-1
\end{array}\right)\left(P_{f} \cdot P_{a}\right)^{c-1}\left\{\left(1-P_{a}\right)+P_{f}\left(1-P_{a}\right)\right\}^{C-c} .
$$

State and Transition Probabilities of the Semi-Markov Model. The state and transition probabilities of the embedded Markov chain, building the basis for the solution of the presented semi-Markov model, are given next. The state probabilities for the idle state $(i=0)$ with respect to the embedded Markov chain are:

$$
p_{0}=p_{0} p_{0}(0)+p_{3(B+1)+1} \cdot p_{4}(0) \text {. }
$$

The first term represents the self-loop within the idle state. That means, that no message is generated during $\tau_{0}=1$ slot corresponding to the probability $p_{0}(0)=e^{-\lambda}$. The second term is related to the transition from the transmission state to the idle state given that no message is generated during $\tau_{4}$. The transition probabilities for the transition from the idle state to the synchronization state are:

$$
p_{0, j}=p_{0}(j)=\left\{\begin{array}{rl}
e^{-\lambda} ; & j=0 \\
1-e^{-\lambda} ; & j=1 \\
0 ; & 2 \leq j \leq(B+1)
\end{array} .\right.
$$

Recall that at most one packet could be generated within a sojourn time of one slot according to the model assumptions.

The synchronization state can be reached from the idle state and the transmission state. Accordingly, the state probabilities for the synchronization state, i.e. $1 \leq i \leq B+1$, are:

$$
p_{i}=\left\{\begin{aligned}
p_{0} p_{0}(1)+p_{3(B+1)+1} \cdot p_{4}(1)+p_{3(B+1)+2} \cdot p_{4}(0) ; & i=1 \\
p_{3(B+1)+1+i} \cdot p_{4}(0)+p_{3(B+1)+i} \cdot p_{4}(1) ; & 2 \leq i \leq B \\
p_{4(B+1)} p_{4}(1) ; & i=B+1
\end{aligned}\right.
$$


The transition probabilities for the transition from the synchronization state to the evaluation state are:

$$
p_{i,(B+1)+j}=\left\{\begin{aligned}
p_{1}(0) ; & 1 \leq i \leq B, j=i \\
p_{1}(1) ; & 1 \leq i \leq B, j=i+1 \\
1 ; & i=j=B+1
\end{aligned}\right.
$$

Note that $p_{2}(0)=e^{-\lambda}$ and $p_{2}(1)=1-e^{-\lambda}$ since at most one message can be generated during $\tau_{1}=1 / 2$. Further, since the transmission buffer cannot accommodate more than $B$ packets, the transition from $p_{B+1}$ to $p_{2(B+1)}$ occurs with probability equal to one.

The evaluation state may be reached from the synchronization state and from the delay state. The corresponding state probabilities for the evaluation state, i.e. $(B+1)+1 \leq i \leq 2(B+1)$, are:

$p_{(B+1)+i}= \begin{cases}p_{1} \cdot p_{1}(0) & ; \quad i=1 \\ p_{i-1} p_{1}(1)+p_{i} p_{1}(0) & ; \quad 2 \leq i \leq B \\ p_{B} p_{1}(1)+p_{B+1}+p_{3(B+1)}+p_{2(B+1)+B} \cdot p_{3}(1) ; & i=B+1\end{cases}$

The transition from the evaluation state to the delay state depends on the blocking probability $P_{B}$, whereas the transition from the evaluation to the transmission state is related to $\left(1-P_{B}\right)$. Thus, the transition probabilities for the transition from the evaluation state to the delay state for $\tau_{2}=1$ are:

$$
p_{(B+1)+i, 2(B+1)+j}=\left\{\begin{aligned}
P_{B} \cdot p_{2}(0) ; & 1 \leq i \leq B, j=i \\
P_{B} \cdot p_{2}(1) ; & 1 \leq i \leq B, j=i+1 \\
P_{B} ; & i=j=B+1 .
\end{aligned}\right.
$$

And the transition probabilities for the transition from the evaluation state to the transmission state for $\tau_{2}=1$ are:

$$
p_{(B+1)+i, 3(B+1)+j}=\left\{\begin{aligned}
\left(1-P_{B}\right) \cdot p_{2}(0) ; & 1 \leq i \leq B, j=i \\
\left(1-P_{B}\right) \cdot p_{2}(1) ; & 1 \leq i \leq B, j=i+1 \\
1-P_{B} ; & i=j=B+1
\end{aligned}\right.
$$

The delay state can only be reached from the evaluation state. As a result, the state probabilities for the delay state, i.e. $2(B+1)+1 \leq i \leq 3(B+1)$, assuming $\tau_{2}=1$, are:

$$
p_{2(B+1)+i}=\left\{\begin{array}{rl}
p_{(B+1)+1} \cdot P_{B} p_{2}(0) ; & i=1 \\
P_{B}\left\{p_{(B+1)+i-1} \cdot p_{2}(1)+p_{(B+1)+i} \cdot p_{2}(0)\right\} ; & 2 \leq i \leq B \\
P_{B}\left\{p_{(B+1)+B} \cdot p_{2}(1)+p_{2(B+1)}\right\} ; & i=B+1
\end{array} .\right.
$$


The transition probabilities for the transition from the delay state to the evaluation state are:

$$
p_{2(B+1)+i,(B+1)+j}=\left\{\begin{aligned}
p_{3}(0) ; & 1 \leq i \leq B, j=i \\
p_{3}(1) ; & 1 \leq i \leq B, j=i+1 \\
1 ; & i=j=B+1
\end{aligned}\right.
$$

Finally, the transmission state is reachable after having been in the evaluation state. The state probabilities for the transmission state, i.e. $3(B+1)+$ $1 \leq i \leq 4(B+1)$, for $\tau_{2}=1$, are:

$p_{3(B+1)+i}=\left\{\begin{aligned}\left(1-P_{B}\right) \cdot p_{(B+1)+1} \cdot p_{2}(0) ; & i=1 \\ \left(1-P_{B}\right)\left\{p_{(B+1)+i-1} \cdot p_{2}(1)+p_{(B+1)+i} \cdot p_{2}(0)\right\} ; & 2 \leq i \leq B \\ \left(1-P_{B}\right)\left\{p_{(B+1)+B} \cdot p_{2}(1)+p_{2(B+1)}\right\} ; & i=B+1\end{aligned}\right.$

The transition probabilities for the transition from the transmission state to the idle and synchronization state are:

$$
p_{3(B+1)+i, j}=p_{4}(j-i+1) ; \quad 1 \leq i \leq B, \quad i-1 \leq j \leq i
$$

Numerical Solution of the Amalytical Mlodel. The required state and transition probabilities for the solution of the presented semi-Markov model are iteratively calculated according to the following computational steps.

(1) Choose initial value for $P_{B}$.

(2) Compute transition and state probabilities $p_{i, j}$ and $p_{i}$ via (14), (16), (18), (19), (21), (23), and via (13), (15), (17), (20), (22), respectively.

(3) Compute state probabilities $P_{i}, i \in\{0,1, \ldots, 4(B+1)\}$ and $S_{s, d}, s, d \in$ $\{1,2, \ldots, M\}$ via (1) and (2), respectively.

(4) Compute $P_{B}$ via (5), (9), (11) and (12).

(5) Check for convergence, i.e., whether $\left|P_{B, \text { old }}-P_{B, \text { new }}\right|<\varepsilon$.

(6) Stop algorithm, when $P_{B}$ has converged, otherwise go to step 2 for the next iteration.

As the initial value for $P_{B}$, we used constantly $P_{B}=0.1$, although the iterative algorithm proved to be almost independent of the initial state. The state space cardinality, generally given by $4(B+1)+1$, corresponds to 45 for $B=10$ resulting in a fairly low computation time. 


\subsection{Model Validation}

In order to validate the developed analytical model, its results are compared to those obtained from computer simulations in this subsection. The simulator is written in $\mathrm{C}++$ and the simulation runs had to achieve a confidence interval of $1 \%$ for a $95 \%$ confidence level. Of course, also in the simulations, a symmetric Poisson arrival process along with a uniform traffic scenario has been assumed and the packet lengths were fixed to one slot. Note that the evaluation (processing) delay within the model is set to $\tau_{2}=T_{p}=1$ in this study. Further, as main performance metrics, the mean queueing delay, measured from the generation instant of a packet to the moment of its complete transmission by the source node, and the throughput per channel, are taken here. The transmission buffer sizes are limited to 10 packets, i.e. $B=10$. Finally, note that nonzero transmitter tuning times (in practice, the tuning time is incorporated by using accordingly larger slot sizes) are not taken into account throughout the following studies since this would only introduce a constant delay overhead at the source nodes without negatively affecting the proper operation of the access schemes.

Fig. 4 shows the mean queueing delay versus the channel throughput normalized to its maximum for 16 and 24 nodes, respectively, while assuming $C=4$. Note that the maximum achievable channel throughput for the uniform traffic scenario is equal to 2 times the medium bit rate in a single unidirectional ring. Both variants, i.e., strategy 1 and 2 (see above), in terms of the RND buffer selection scheme have been considered.

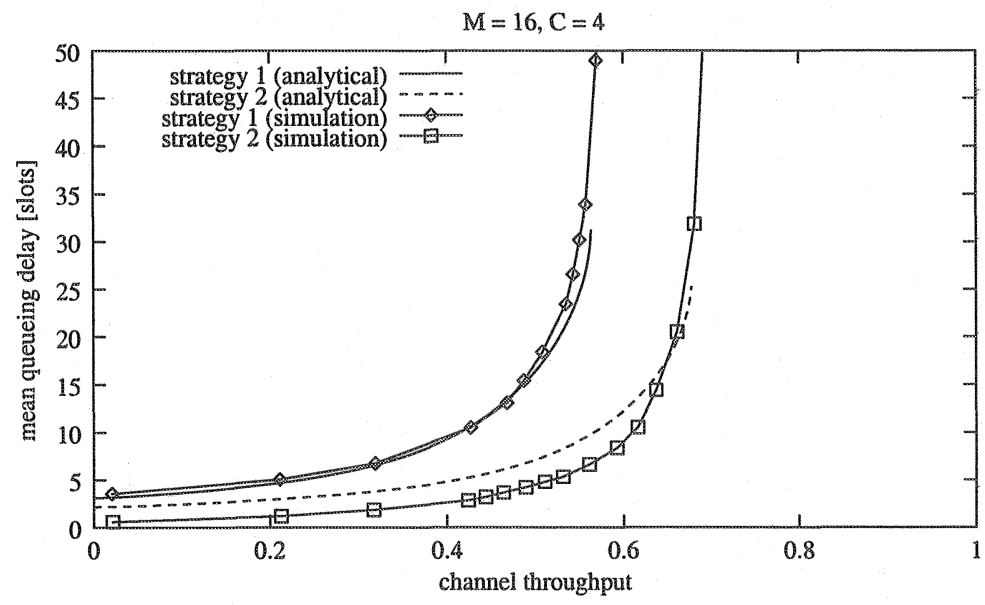

Figure 4. Comparison of analytical and simulation results for $D=4$ and $B=10$.

As can be seen in Fig. 4, the simulation results match very well with the analytical results obtained from the semi-Markov model. Particularly, the sat- 
uration effects can be perfectly predicted making time-consuming and cumbersome simulations unnecessary for capturing the general behavior of the analyzed RND access scheme. Another observation which can be made is that strategy 2 appears to be much more efficient than strategy 1 , in accordance with our expectations. Additionally, due to its ease of implementation, it is highly preferable over the other scheme. Therefore, we only refer to strategy 2 in conjunction with the RND access mechanism in the sequel.

\subsection{Simulation Results}

In order to evaluate the performance of various selection strategies for more realistic traffic conditions, extensive simulations have been conducted. The assumptions for the simulations are almost the same as in Subsection 4.2 with the following additions. Besides the Poisson arrival processes with mean arrival rate $\lambda$ per node given in packets/slot, negative-exponentially distributed variable-length packets with a mean length of $L=25$ slots are assumed in the following study. Furthermore, the ring length corresponds to $n=150$ slots accommodating equidistantly spaced nodes, while the length of the slots is set to 1000 bits, yielding a slot duration of $400 \mathrm{~ns}$ for a channel transmission rate of $2.5 \mathrm{Gbit} / \mathrm{s}$. Additionally, we assume that each transmission queue has an almost infinite capacity $(B \rightarrow \infty)$ in order to avoid packet losses at the source nodes due to buffer overflows.

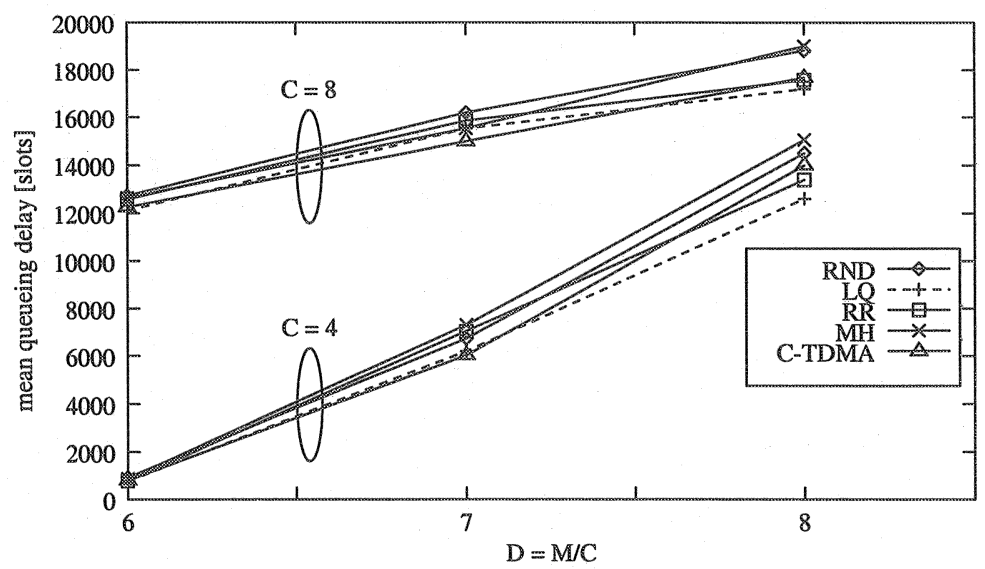

Figure 5. Performance comparison of the buffer selection strategies in dependence of $D$ for $C=4$ and $C=8$ under overload conditions $(n=150$, uniform Poisson traffic, $L=25$, $B \rightarrow \infty)$.

In Fig. 5, the mean queueing delays are plotted against the number of nodes (since the channel number is kept constant to $C=4$ and $C=8$, respectively, here) for the different selection strategies under overload traffic conditions. 
While for smaller numbers of nodes corresponding to lower throughputs, the delays behave rather similarly for the assumed node traffic of $\lambda=0.009$, the LQ scheme provides the best delay performance compared to the other schemes. This primarily results from the achieved load balance among the node's transmission queues (leading to higher throughputs as well). On the other hand, the MH strategy leads to the worst results under overload conditions. This can be intuitively explained by the fact that the channels remain longer busy under heavy network load due to the maximum hop preference at the source nodes, aggravating the immediate access to the medium. Tables 1,2 and Tables 3, 4 show the numerical performance values in more detail for $C=4$ and $C=8$, respectively. As a result, it can be stressed that the RND scheme leads to a satisfactory tradeoff between implementation complexity (costs) and performance, particularly for non-heavy traffic load.

Table 1. Performance comparison for $C=4$ (maximum throughput $=8$ ) and different numbers of nodes related to Figure 5 with $\lambda=0.009$.

\begin{tabular}{c||cc|cc|cc}
\hline \multicolumn{1}{|c||}{} & \multicolumn{2}{c|}{$D=3$} & \multicolumn{2}{c|}{$D=4$} & \multicolumn{2}{c}{$D=5$} \\
\hline strategy & thr. & delay & thr. & delay & thr. & delay \\
\hline \hline RND & 2.71 & 88 & 3.57 & 141 & 4.52 & 269 \\
\hline LQ & 2.71 & 95 & 3.60 & 154 & 4.52 & 279 \\
\hline RR & 2.68 & 86 & 3.59 & 143 & 4.47 & 263 \\
\hline MH & 2.70 & 89 & 3.61 & 150 & 4.43 & 277 \\
\hline C-TDMA & 2.71 & 89 & 3.59 & 144 & 4.46 & 268 \\
\hline
\end{tabular}

Table 2. Performance comparison for $C=4$ (maximum throughput $=8$ ) and different numbers of nodes related to Figure 5 with $\lambda=0.009$.

\begin{tabular}{c||cc|cc|cc}
\hline \multicolumn{1}{c||}{} & \multicolumn{2}{c|}{$D=6$} & \multicolumn{2}{c|}{$D=7$} & \multicolumn{2}{c}{$D=8$} \\
\hline strategy & thr. & delay & thr. & delay & thr. & delay \\
\hline \hline RND & 5.39 & 931 & 5.92 & 6738 & 6.03 & 14505 \\
\hline LQ & 5.36 & 772 & 6.03 & 6202 & 6.15 & 12611 \\
\hline RR & 5.35 & 768 & 5.95 & 7050 & 6.10 & 13396 \\
\hline MH & 5.35 & 870 & 5.91 & 7312 & 6.04 & 15046 \\
\hline C-TDMA & 5.36 & 807 & 5.97 & 6027 & 6.08 & 13998 \\
\hline
\end{tabular}


Table 3. Performance comparison for $C=8$ (maximum throughput $=16$ ) and different numbers of nodes related to Figure 5 with $\lambda=0.018$.

\begin{tabular}{c||cc|cc|cc}
\hline \multicolumn{1}{c||}{} & \multicolumn{2}{c|}{$D=3$} & \multicolumn{2}{c|}{$D=4$} & \multicolumn{2}{c}{$D=5$} \\
\hline strategy & thr. & delay & thr. & delay & thr. & delay \\
\hline \hline RND & 10.54 & 1564 & 11.82 & 6836 & 12.68 & 9384 \\
\hline LQ & 10.63 & 970 & 12.23 & 6112 & 12.87 & 9649 \\
\hline RR & 10.59 & 1604 & 11.90 & 6456 & 12.67 & 9852 \\
\hline MH & 10.36 & 2190 & 11.71 & 6771 & 12.56 & 9814 \\
\hline C-TDMA & 10.63 & 1039 & 12.16 & 5977 & 12.78 & 9865 \\
\hline
\end{tabular}

Table 4. Performance comparison for $C=8$ (maximum throughput $=16$ ) and different numbers of nodes related to Figure 5 with $\lambda=0.018$.

\begin{tabular}{c||cc|cc|cc}
\hline \multicolumn{1}{c||}{} & \multicolumn{2}{c|}{$D=6$} & \multicolumn{2}{c|}{$D=7$} & \multicolumn{2}{c}{$D=8$} \\
\hline strategy & thr. & delay & thr. & delay & thr. & delay \\
\hline \hline RND & 13.09 & 12734 & 13.35 & 16197 & 13.43 & 18819 \\
\hline LQ & 13.26 & 12113 & 13.45 & 15529 & 13.72 & 17199 \\
\hline RR & 13.08 & 12582 & 13.30 & 15851 & 13.50 & 17576 \\
\hline MH & 13.10 & 12642 & 13.29 & 15529 & 13.52 & 19004 \\
\hline C-TDMA & 13.21 & 12255 & 13.39 & 14985 & 13.66 & 17676 \\
\hline
\end{tabular}

\section{Conclusions}

In this paper, a comprehensive analytical model based on the semi-Markov modelling theory is developed for examining the performance of an access protocol for a slotted WDM metro ring network which is based on a-posteriori random (RND) access scheme. The considered protocol is analyzed with respect to throughputs per channel and mean packet queueing delays for uniform traffic. In terms of the underlying node architecture, each node is equipped with one tunable transmitter and one fixed-tuned receiver, while the subcarrier multiplexing technique is assumed for delivering the required signaling information. Alternatively, a dedicated optical control channel could be employed as well for signaling at the expense of an additional fixed transceiver per node. The RND selection scheme is found to provide a satisfactory compromise between performance and implementational complexity compared to different buffer selection mechanisms in conjunction with the a-posteriori access strategy. 


\section{References}

[1] K. Bengi. Optical Packet Access Protocols for WDM Networks. Kluwer Academic Publishers, 2002.

[2] K. Bengi and H.R. van As. Efficient QoS support in a slotted multi-hop WDM metro ring. IEEE Journal on Selected Areas in Communications (special issue on "WDM-Based Network Architectures"), 20(1):216-227, Jan. 2002.

[3] A. Bianco, V. Distefano, A. Fumagalli, E. Leonardi, and F. Neri. A-posteriori access strategies in all-optical slotted WDM rings. Proceedings of GLOBECOM'98, Sydney, Australia, pages 300-306, Nov. 1998.

[4] I. Chlamtac, A. Fumagalli, L.G. Kazovsky, and P.T. Poggiolini. A Multi-Gbit/s WDM optical packet network with physical ring topology and multi-subcarrier header encoding. Proceedings of ECOC'93, Montreux, Switzerland, pages 121-124, Sept. 1993.

[5] J. Fransson, M. Johansson, M. Roughan, L. Andrew, and M.A. Summerfield. Design of a medium access control protocol for a WDMA/TDMA photonic ring network. Proceedings of GLOBECOM'98, Sydney, Australia, pages 307-312, Nov. 1998.

[6] A. Fumagalli, J. Cai, and I. Chlamtac. A token based protocol for integrated packet and circuit switching in WDM rings. Proceedings of GLOBECOM'98, Sydney, Australia, pages 2339-2344, Nov. 1998.

[7] A.E. Kamal and G.K. Janssens. Design, performance and wavelength assignment of a wavelength division multiaccess protocol for optical fibre ring networks. 31(22):23912410, Nov. 1999.

[8] M. Ajmone Marsan, A. Bianco, E. Leonardi, M. Meo, and F. Neri. MAC protocols and fairness control in WDM multirings with tunable transmitters and fixed receivers. IEEE/OSA Journal of Lightwave Technology, 14(6):1230-1244, June 1996.

[9] M. Ajmone Marsan, A. Bianco, E. Leonardi, M. Meo, and F. Neri. On the capacity of MAC protocols for all-optical WDM multi-rings with tunable transmitters and fixed receivers. Proceedings of INFOCOM'96, San Francisco, CA, pages 1206-1216, March 1996.

[10] M. Ajmone Marsan, E. Leonardi, M. Meo, and F. Neri. Modelling slotted WDM rings with discrete-time Markovian models. 32(5):599-615, May 2000.

[11] S. Ross. Stochastic Processes. John Wiley \& Sons, 1983.

[12] I.M. White, D. Wonglumsom, K. Shrikhande, S.M. Gemelos, M.S. Rogge, and L.G. Kazovsky. The architecture of HORNET: A packet-over-WDM multiple-access optical metropolitan area ring network. 32(5):587-598, May 2000. 